UR-1410

ER-40685-857

hep-th/9502056

Dec. 1994

\title{
ON THE COVARIANTIZATION OF THE CHIRAL CONSTRAINTS 1
}

\author{
E.M.C. de Abreu and C.Neves \\ Instituto de Física \\ Universidade Federal do Rio de Janeiro \\ Rio de Janeiro \\ Brasil \\ Clovis Wotzasek? \\ Department of Physics and Astronomy \\ University of Rochester \\ Rochester, NY 14627 \\ $U S A$
}

\begin{abstract}
We show that a complete covariantization of the chiral constraint in the FloreaniniJackiw necessitates an infinite number of auxiliary Wess-Zumino fields otherwise the covariantization is only partial and unable to remove the nonlocality in the chiral boson operator. We comment on recent works that claim to obtain covariantization through the use of Batalin-Fradkin-Tyutin method, that uses just one Wess-Zumino field.
\end{abstract}

\footnotetext{
${ }^{1}$ This work is supported by CNPq, Brasília, Brasil

${ }^{2}$ Permanent address: Instituto de Física, Universidade Federal do Rio de Janeiro, Brasil
} 
The quantization of chiral boson in two-dimensions is a very interesting theoretical problem, which has appeared originaly in the investigation of heterotic string [1], and became quite important in the study of fractional quantum Hall efect [2]. This problem has a simple solution in the Hamiltonian language while it has been beset with enormous difficulties in the Lagrangian side. One of these problems is the covariantization of the second-class chiral constraint, i.e., the transformation from second to first-class, which is the object of investigation in this paper.

The usual Lagrangian route to chiral bosonization starts with a scalar field and projects out one of the chiral components by means of the chiral constraint $\partial_{ \pm} \phi \approx 0$. According to Dirac's theory of constrained systems [3], this is a second- class constraint, but in order to avoid the Lagrange multiplier to become dynamical, one needs it to be first-class. There has been two main routes to the covariantization of the chiral constraint. Siegel [4] proposed to set to zero one component of the energymomentum tensor resulting an action with the chiral constraint squared, which has a reparametrization invariance called as Siegel symmetry. In the quantum level, however, Siegel symmetry becomes second-class again on account of the central extension of the conformal algebra of the energy-momentum tensor [5]. A simple solution to the anomaly problem was given by Hull with the introduction of a new set of auxiliary fields called as no-movers [6]. Besides the anomaly problem, to produce first-class constraints by squaring second-class constraints has been criticized [7]. This sort of primary constraint does not produce the complete set of constraints when Dirac's algorithm is employed, and is (infinitely) reducible.

The second route to covariantization starts with the Floreanini-Jackiw model [8] which is a singular theory from Dirac's point of view, the resulting constraint being the second-class chiral constraint. The constraint's nature is changed à la FaddeevShatashvili 9] with the introduction of Wess-Zumino auxiliary fields. The covariantization of the chiral constraint in this route has been proposed in two conceptually differents papers: in Ref. [10], an infinite family of scalar fields, coupled by a combination of right and left chiral constraints carefully adjusted to be first-class from the start, was shown to have a single chiral boson in the spectrum by use of very elegant group theoretical methods. In Ref. [11], the FJ chiral boson was iteratively changed to modify the nature of chiral constraint to render it first-class. Following this route the modified Floreanini-Jackiw model with the original chiral field plus the set of Wess-Zumino fields will always have one left over second-class constraint that must as well be converted into first-class if a complete covariantization is desired. It is worth of mention that, as explained by Boyanovsky 12], the inclusion of constraint conversion terms do not change the physical spectrum of the theory but only the 
unphysical sector of the Hilbert space. Therefore the chiral boson spectrum which we begin with will not be changed during the constraint conversion process. For the case of FJ-chiral boson, the algorithm in [11] revealed the necessity of an infinite number of auxiliary fields, thus stablishing a connection with the model in [10]. This necessity of an infinite set of auxiliary fields in the the covariantization process of the chiral boson operator can be traced back to the fact that the operator of the chiral boson action is the square root of the D'Lambertian operator, therefore of nonlocal nature. Consequently, in following this route we are basically exchanching the non-locality by auxiliary fields, as we are used to. The equivalence of proposals [10 and [11 has been proved in [13]. We mention that the use of infinite auxiliary fields in the covariantization process of second-class constraints has also been used by Mikovic et al [14] in the context of the super-particle quantization.

It is our intention in this paper to work out the possibility of stopping the constraint conversion process, mentioned above, at any (finite) stage, say after $\mathrm{N}$ iterations, even if a second-class constraint remains, and study its consequences. It is expected that in doing this we would destroy the possibility of obtaining the complete Wess-Zumino Lagrangian, and the theory would only be partially covariantized. Furthermore, one should not expect to completely remove the non-locality of the chiral boson operator by stopping the constraint conversion process at any finite $\mathrm{N}$, i.e., with use of a finite number of auxiliary fields. We will see that these expectations are indeed fullfilled and in doing so we get a theory that is neither fully covariant nor local. We plan to stop the constraint conversion process in two different ways and verify their equivalence. Firstly we will follow a constraint conversion method introduced by one of us some years ago in the context of the chiral Schwinger model [15. As explained there, this method is unable to provide the complete Wess-Zumino Lagrangian, but corresponds only to some gauge-fixed version of it. Putting in another words, this procceedure only restores part of the symmetry lost during the quantization process, and this happens due to the existence of a "stopping rule" that stops the covariantization process even if a second-class constraint is still present (see below). Another way of doing it is by a simple elimination of the left over second-class constraint using Dirac brackets for the variables involved. This can be done after an arbitrary $\mathrm{N}$ number of steps. It will then be clear that the results of the first method corresponds to choose to stop at $\mathrm{N}=1$ in the second method. At this point we make contact with a third proposal for the covariant quantization of FJ chiral boson which has recently appeared in the literature 16, 17] (see also [23]). Differently of the other two schemes, these works claim to make use of a single Wess-Zumino auxiliary field to produce the constraint conversion into first-class. In [16, 17], use is made of the 
constraint conversion technique proposed by Fradkin and colaborators $[18$. The basic idea in [18] is to produce the change in the constraint's nature, by enlarging the Hamiltonian phase-space with ghosts and Lagrange multipliers of opposite statistics in order to obtain the nilpotency of the BRST charge.

We show that the results we found using both methods discussed above are equivalent to [16, 17] in the sense that the covariantization of the initial chiral constraint is acchieved with only one auxiliar field. From these results we conclude that this third proposal for covariantization with only one Wess-Zumino field is only able to acchive partial covariantization, and is equivalent to stopping the iterative method of [11] at the $\mathrm{N}=1$ stage.

To begin with, let us review the basic idea proposed in [15]. Suppose a noninvariant theory, described by $2 \mathrm{n}$ phase-space variables, a Hamiltonian $H_{0}$, and a set of $2 \mathrm{~m}$ second-class constraints $\Omega_{a}$. The Dirac matrix of the constraints is defined as

$$
C_{a b}=\left\{\Omega_{a}, \Omega_{b}\right\}
$$

and satisfy $\operatorname{det} C_{a b} \neq 0$ due to the second-class nature of the constraints. Call $H=$ $H_{0}+\lambda_{a} \Omega_{a}$ the total or Dirac Hamiltonian. Since there is no avaiable symmetry, by assumption, the Lagrange multipliers $\lambda_{a}$ are not arbitrary and can be determined from the consistency on the time development of $\Omega_{a}$. Next, we introduce the auxiliary Wess-Zumino fields $\theta_{a}$, which are choosen to satisfy

$$
\left\{\theta_{a}, \theta_{b}\right\}=C_{a b}^{-1}
$$

It is then easy to verify that the new set of constraints $\tilde{\Omega}_{a}$, defined by

$$
\Omega_{a} \rightarrow \tilde{\Omega}_{a}=\Omega_{a}+\sum_{b} C_{a b} \theta_{b}
$$

is first-class $\left\{\tilde{\Omega}_{a}, \tilde{\Omega}_{b}\right\}=0$. The new set of constraints may, however, necessitates a secondary constraint manifold to guarantee its time development stability, and it is possible that some of these secondary constraints may be second-class again. In this eventuality a new set of Wess-Zumino fields would become necessary and so on. In order to avoid such behavior, we introduce a "stopping rule" by demanding the firstclass set of constraints (3) to commute with a generalized Hamiltonian $\tilde{H}$, obtained from the original one, as a (functional) Taylor series of the Wess-Zumino fields

$$
H \rightarrow \tilde{H}=H_{0}+\sum_{a} M_{a} \theta_{a}+\frac{1}{2} \sum_{a b} M_{a b} \theta_{a} \theta_{b}+\ldots
$$


The "stopping rule" mentioned above takes the form

$$
\left\{\tilde{\Omega}_{a}, \tilde{H}\right\}=0
$$

Solving equation (5), order by order in $\theta$, fixes the expansion coeficient as

$$
\begin{gathered}
M_{a}^{(1)}=\left\{H_{0}, \Omega_{a}\right\} \\
M_{a b}^{(2)}=\left\{M_{a}^{(1)}, \Omega_{b}\right\}
\end{gathered}
$$

and so on, as explained in 15$]$.

Let us consider now the case of Floreanini-Jackiw chiral boson. The FloreaniniJackiw [8] chiral boson theory is a first order model with the spectrum being derived directly from its equation of motion, without the necessity of external constraints. The drawback of this formulation is the lack of explicit Lorentz covariance, which makes the coupling with gauge or gravitation fields rather difficult [19, 20, 21, 22]. For the case of the (left mover) FJ chiral boson, described by

$$
L=\int d x\left(\dot{\phi} \phi^{\prime}-\phi^{\prime 2}\right)
$$

the Dirac Hamiltonian reads

$$
\begin{gathered}
H=\int d x\left[\phi^{2}+\lambda\left(\pi-\phi^{\prime}\right)\right] \\
=\int d x \pi \phi^{\prime}
\end{gathered}
$$

where $\pi=\delta \mathcal{L} / \delta \dot{\phi}$ is the canonical momentum of $\phi(x)$ and $\lambda=\phi^{\prime}$ has been determined from the consistency condition for the evolution of the chiral constraint $\Omega=\pi-\phi^{\prime}$. The modified (first-class) constraint is, according to definition (3), given by

$$
\Omega \rightarrow \tilde{\Omega}=\pi-\phi^{\prime}-2 \theta^{\prime}
$$

where $\theta$ is the Wess-Zumino field, satisfying condition (2)

$$
\left\{\theta^{\prime}(x), \theta^{\prime}(y)\right\}=\frac{1}{2} \delta^{\prime}(x-y)
$$

which shows that this auxiliary field is itself of chiral nature. The other brackets are canonical 


$$
\begin{aligned}
& \{\phi(x), \pi(y)\}=\delta(x-y) \\
& \{\phi(x), \theta(y)\}=0 \\
& \{\pi(x), \theta(y)\}=0
\end{aligned}
$$

To compute $\tilde{H}$ is now a pretty simple algebraic work. Using (6) we obtain

$$
\begin{gathered}
M^{(1)}(x)=-2 \phi^{\prime \prime}(x) \\
M^{(2)}(x, y)=-2 \delta^{\prime \prime}(x-y)
\end{gathered}
$$

while all the remaing coefficients vanish. Then, using (12) into (8) we get

$$
\tilde{H}=\int d x\left(\phi^{\prime 2}+2 \phi^{\prime} \theta^{\prime}+\theta^{2}\right)=\int d x\left(\phi^{\prime}+\theta^{\prime}\right)^{2}
$$

Notice that making use of (10) and (11) we obtain a first-class algebra for the (modified) chiral constraint

$$
\{\tilde{\Omega}(x), \tilde{\Omega}(y)\}=0
$$

We have thus succeeded in turning into first-class the original second-class constraint of the Floreanini-Jackiw model. To make contact with the results in Ref. [16, 17, we observe that a concrete realization the algebra (10) in terms of a canonically conjugated pair is given by

$$
\theta^{\prime}=\frac{1}{2} \xi+\frac{1}{2} \eta^{\prime}
$$

with

$$
\{\eta(x), \xi(y)\}=\delta(x-y)
$$

In this representation, the canonical Hamiltonian (13) and the first-class chiral constraint become

$$
\begin{gathered}
\tilde{H}=\int d x\left(\phi^{\prime}+\frac{1}{2} \xi+\frac{1}{2} \eta^{\prime}\right)^{2} \\
\tilde{\Omega}=\pi-\phi^{\prime}-\xi-\eta^{\prime}
\end{gathered}
$$


which are the results of [16, 17]. Quite clearly this first-class chiral constraint (18) is a combination of a right and a left chiral constraint

$$
\tilde{\Omega}=\Omega^{(-)}-\Omega^{(+)}
$$

where the chiral constraints $\Omega^{( \pm)}(x)$ satisfy two uncoupled Kac-Moody algebras

$$
\begin{gathered}
\left\{\Omega^{( \pm)}(x), \Omega^{( \pm)}(y)\right\}=(\mp) 2 \delta^{\prime}(x-y) \\
\left\{\Omega^{(+)}(x), \Omega^{(-)}(y)\right\}=0
\end{gathered}
$$

Notice that the constraint (18) is of the same sort as that introduced in Refs. 110 and 111] showing that some connection might exist between these results. It is precisely this relationship that we intent to clarify below.

In the sequence we want to show that the above result, Eqs.(9) and (13) (or (17) and (18)) can be obtained as a particularization of the method given in Ref. [11] if, after one iteration, the emergent second-class constraint instead of being covariantized is strongly resolved à la Dirac. To this end we quickly review the basic points of [11, taking the opportunity to present a new approach to that problem. Indeed, the results of 111 after $\mathrm{N}$ iterations, are easily found if in the FJ Lagrangian (7) we make the substitution

$$
\phi_{0} \rightarrow \phi_{0}+\phi_{1}+\ldots+\phi_{N}
$$

where $\phi_{0}$ represents the original chiral boson field $\left(\phi \rightarrow \phi_{0}\right)$ and the remaining $\phi_{k}$ are $\mathrm{N}$ auxiliary Wess-Zumino fields. Introducing (21) into (7) gives

$$
L^{(0)} \rightarrow L^{(N)}=\sum_{k=0}^{N} \int d x\left(\dot{\phi}_{k} \phi_{k}^{\prime}-\phi_{k}^{\prime 2}\right)+\sum_{m=k+1}^{N} \sum_{k=0}^{N-1} \int d x\left(\dot{\phi_{k}}-\phi_{k}^{\prime}\right) \phi_{m}^{\prime}
$$

which is the result in [11]. Consequently, the original chiral constraint $\Omega_{0}$ present in the Floreanini-Jackiw action is transformed into a set of $(N+1)$ constraints

$$
\begin{gathered}
\Omega_{0} \rightarrow\left\{\Omega_{0}, \Omega_{1}, \ldots, \Omega_{N}\right\} \\
\Omega_{k} \approx 0
\end{gathered}
$$


Explicitly, these constraints read

$$
\begin{gathered}
\Omega_{N}=\pi_{N}-\phi_{N}^{\prime} \\
\Omega_{k}=\pi_{k}-\phi_{k}^{\prime}-2 \sum_{m=k+1}^{N} \phi_{m}^{\prime}
\end{gathered}
$$

and the canonical Hamiltonian becomes

$$
H \rightarrow H_{N}=\int d x \sum_{m, n=0}^{N} \phi_{m}^{\prime} \phi_{n}^{\prime}
$$

which is easily obtained from $\mathcal{H}_{0}=\phi^{\prime 2}$ using the substitution (21). As discussed above, the last constraint $\Omega_{N}$ always break the emergent symmetry by keeping the second-class character of the iterated theory.

Suppose we decide to stop the iteration at this point. This can be done by explicitly solving the left over second-class constraint $\Omega_{N}(x) \approx 0$. This is possible as long as we use the corresponding Dirac bracket for the variables involved. The relevant Dirac bracket in this case is, from (24)

$$
\left\{\phi_{N}, \phi_{N}\right\}^{*}=\frac{1}{2} \delta^{\prime}(x-y)
$$

and the remaining variables satisfy

$$
\left\{\phi_{k}, \phi_{m}\right\}^{*}=0
$$

In particular, let us consider the partial covariantization scheme above for the case $N=1$. We have then

$$
\left\{\phi_{1}, \phi_{1}\right\}^{*}=\frac{1}{2} \delta^{\prime}(x-y)
$$

and

$$
\left\{\phi_{0}, \phi_{0}\right\}^{*}=0
$$

The canonical Hamiltonian reads

$$
H_{1}=\int d x\left(\phi_{0}^{\prime}-\phi_{1}^{\prime}\right)^{2}
$$


and the chiral constraint becomes

$$
\Omega_{0}=\pi_{0}-\phi_{0}^{\prime}-2 \phi_{1}^{\prime}
$$

If we relabel the fields as $\phi_{0} \rightarrow \phi$ and $\phi_{1} \rightarrow \theta$, we reobtain the results above (Eqs.(9) and (13)) therefore justifying our claiming that those schemes are only able to provide a partial covariantization for the chiral boson theory.

In summary, we have worked the problem of covariantization of the bosonic chiral constraint following the methodology of Ref. [15]. Differently from the approach of 11] where an infinite number of auxiliary fields is necessary, this one only needs one Wess-Zumino field. This result was shown to agree with with a paper by Ghosh 16, 17 using 18. We have then showed that the covariantization with just one Wess-Zumino auxiliary field is incomplete and corresponds to stopping the iterative process of [11] after a single iteration, through the (strong) resolution of the left over chiral constraint à la Dirac. This seem to be an expected result since the nonlocality of the chiral boson operator points for the necessity of an infinite set of auxiliary fields as found in [11. It is not clear for us at the present if this results are indicating an essential difficulty in the formalism of Ref. [15] and [18] or are just accidental and restricted to this (uncommom) model. Work in this direction is in progress.

\section{References}

[1] D.J.Gross, J.A.Harvey, E.Martinec, R.Rhom, Phys.Rev.Lett.54 (1985)502.

[2] See, for instance, "Quantum Hall Effect", M.Stone ed. (World Scientific 1992).

[3] P.A.M.Dirac, Lectures on Quantum Mechanics (Yeshiva University Press, New York, N.Y.) 1964.

[4] W.Siegel, Nucl.Phys.B238(1984)307.

[5] R.Nepomechie , L.Mezincescu, Phys.Rev.D37 (1988) 3067 and A.Schwimmer , C. Imbimbo, Phys. Lett.B193 (1987) 455.

[6] C.Hull, Phys.Lett.B206(1988)234.

[7] B.McClain and Y.S.Wu, University of Utah preprint/1988 and M.Lledó and A.Restuccia, USB preprint/1990. 
[8] R.Floreanini, R.Jackiw, Phys.Rev.Lett.59 (1987) 1873.

[9] L.D.Faddeev and L.Shatashvili, Phys.Lett.B167(1986)225.

[10] B.McClain, Y.S.Wu, F.Yu, Nucl.Phys.B343(1990)689.

[11] C.Wotzasek, Phys.Rev.Lett.66(1991)129-133

[12] D. Boyanovsky, J. Schmidt and M. F. L. Golterman, Ann. Phys. (NY) 185 (1988) 111.

[13] C.Wotzasek, Phys. Rev. D44 (1991) R1623.

[14] A.Mikovic, M.Rocek, W.Siegel, P. van Nieuvenhuizen, J.Yamron, and A.E. van de Ven, Phys. Lett. B235 (1990) 106.

[15] C.Wotzasek, Intl.J.Mod.PhysA5(1990)1123.

[16] Subir Ghosh, Phys. Rev. D49 (1994) 2990.

[17] Subir Ghosh, Mod. Phys. Lett. A9 (1994) 535.

[18] I.A.Batalin and E.S.Fradkin, Nucl.Phys.B279(1987)514 and I.A.Batalin and I.V.Tyutin, Intl.J.Mod.Phys.A6(1991)3255.

[19] J.Sonenschein, Nucl.Phys.B309(1988)752.

[20] S. Bellucci, R. Brooks and J. Sonnenschein, Nucl. Phys. B304 (1988) 173 and S. Bellucci, M. F. L. Golterman and D. N. Petcher, Nucl. Phys. B326 (1989) 307.

[21] F.Bastianelli and P.Van Nieuwenhuizen, Phys.Lett.B217 (1989) 98.

[22] K.Harada,Phys.Rev.Lett.64(1990)139.

[23] After the completion of this work we became aware of the paper by R.Amorim and J.Barcelos-Neto, "BFT quantization of the FJ chiral boson", IF-UFRJ$13 / 94$, on the covariantization of the chiral constraint using the Batalin-Fradkin and Tyutin technique for constraint conversion. In this paper they were able to show that the Lagrangian for the converted theory results being nonlocal, as expected. We thank R.Amorim for making a copy of that work avaiable for us prior to its publication. 